\title{
تقويم أنثطة التواصل الشفوي/السمعي في سلسلة كتب العربية الاتصالية من وجهة نظر المعلمين
}

\author{
رجا فبريان ,جمال عبد الناصر زكريا

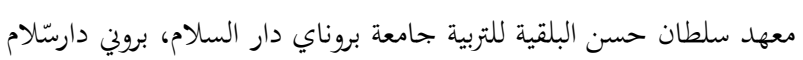 \\ rojjap@yahoo.com
}

\section{ملخّصص}

هدف هذا البحث إلى تقويم أنشطة التواصل الشفوي/السمعي في سلسلة كتب العربية الاتصالية المقررة

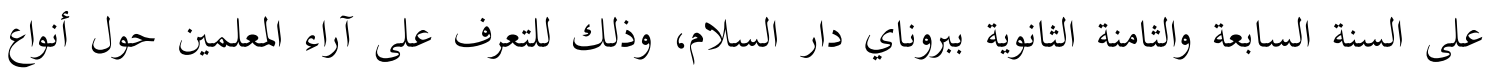

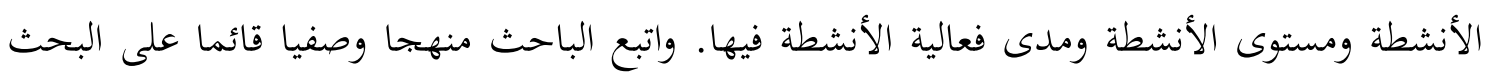

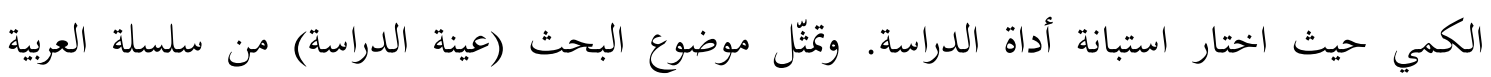

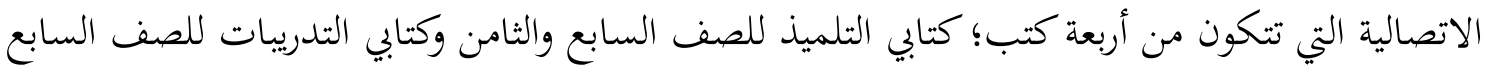

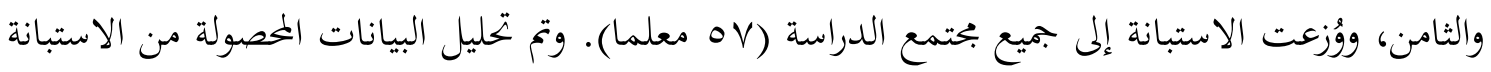

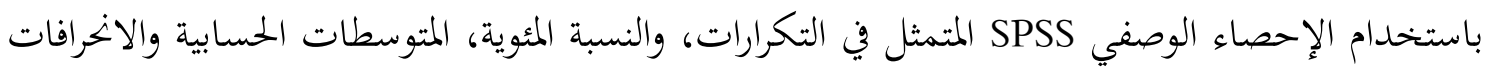

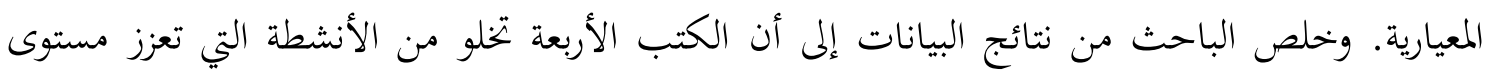

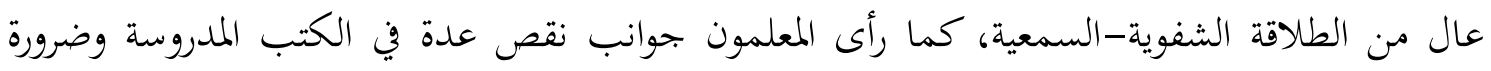
إعادة النطر فيها.

\section{مدخل}

يقتضي التطوير التربوي القيام بعمليات تقويم الكتب ومراجعها للوقوف على جوانب القوة لتعزيزها

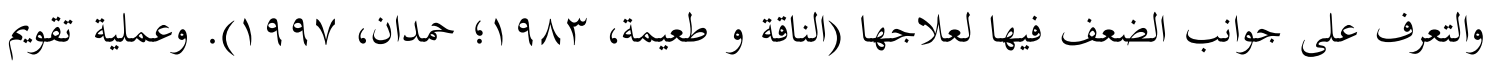

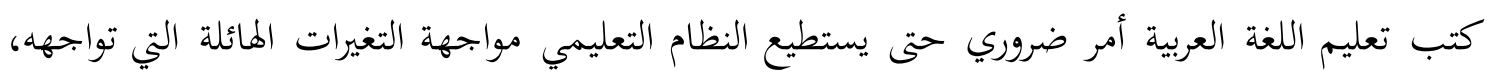

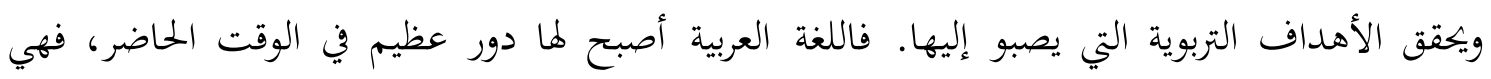

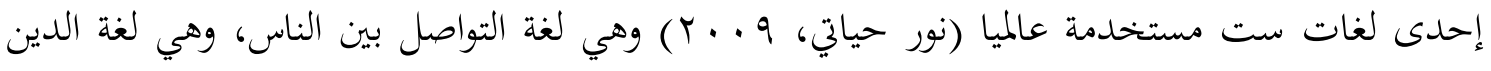
على وجه الخصوص. 
ومن خصائص الكتاب الجيد تضمنه للأنشطة التعليمية المناسبة، حيث تمثل الأنشطة القلب النابض لها دور كبير في تشكيل خبرات المتعلم وتعديل أنماط سلوكه (الهاشمي ومحسن، و ج. . ب). فالأنشطة

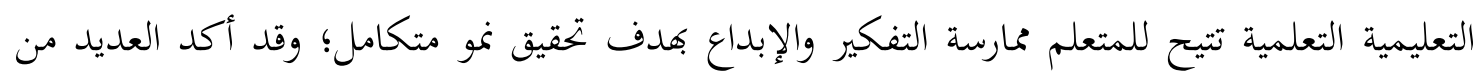

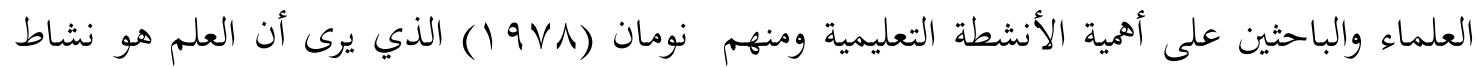
يمارسه المتعلم وأنه ليس بحموعة حقائق يكتسبها المتعلم يستحضرها في اختبار تحريري كما أها ليست سلسلة بحارب ينبغي أداؤها في المختبر فقط. وهذه الأنشطة بنسبة كتاب اللغة العربية تزداد أهميتها لتشكيل مهارات المتعلمين اللغوية.

لقد بدأت دراسة اللغة العربية في المدارس الثانوية التابعة لوزارة التربية منذ عام ب99 ام كمادة اختيارية، وتُدرس لمتعلمي السنة السابع والثامن، وتقوم إدارة تطوير المناهج بوزارة التربية في سلطنة بروناي دار السلام، بتطوير المناهج التعليمية وتحديثها، في ضوء النظام التعليمي في القرن الحادي والعشرين كت Century) تتكون من كتاب التلميذ وكتاب التدريبات للصف السابع وكتاب التلميذ وكتاب التدريبات للصف الثامن. لقد أصدرها قسم تطوير المناهج بوزارة التربية بروناي دار السلام ليتمّ استعمالها ككتب المقررة في المدارس وكسباب الثانوية ببروناي دار السلام.

تقف هذه الدراسة على تقويم أنشطة التواصل الشفوي-السمعي في سلسلة كتب العربية الاتصالية للصف السابع والثامن ، نظرا لما للتواصل الشفهي من أهمية في تعليم اللغة العربية لدى متعلمي المدارس الثانوية بالسلطنة بروناي دار السلام. ويمثّل تقويم أنشطة سلسلة كتب العربية الاتصالية للصف الصف السابع والثامن أهيمة تربوية، نظرا لما تمثله هتان السنتان من مكانة مهمة حيث إفما مرحلة تعليمية مهمة في نظام التعليم البروناوي

\section{مشكلة البحث}

لا تزال الكتب المدرسية تلعب دورا هاما في عملية التعليم والتعلم وعلى الرغم من آثار

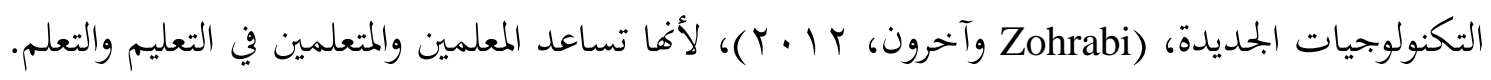
وفي اللغة العربية، توفّر الكتب المدرسية المواد التعليمية التي تساعد المتعلمين على إتقان اللغة العربية

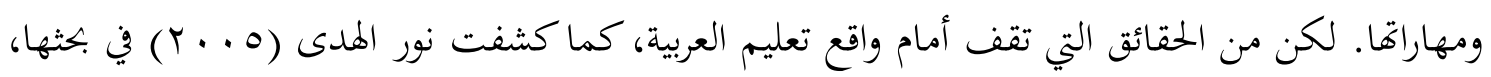
كثرة الشكاوى من أخطاء الدارسين الملايويين المقبلين على تعلم اللغة العربية، وكانت من أسبابها تعليما مضمون المقرر الذي لا يكون مناسبا لهم. ووجد الباحث بعض البحوث العلمية قد تناولت بعض قضايا تعليم اللغة

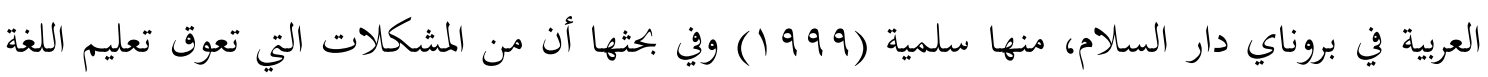

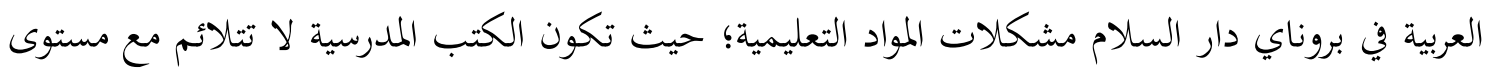


التلاميذ، كما أن محتوياها المستخدمة في التعليم غير واضحة وأسلوبها صعب، وشكلها الفني غير جذاب.

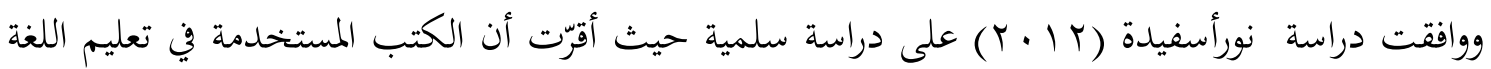

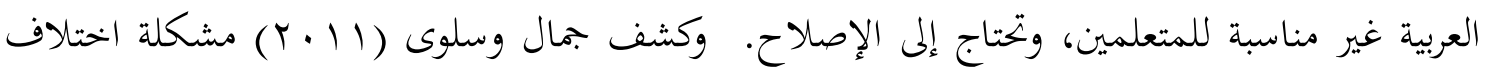
مقرر اللغة العربية المستخدم في تعليم اللغة العربية بالمدارس الثانوية، حيث وجدا استخدام المقررات من معند تأليف فرق أو بحموعة من المدرسين، وليس المقرر أو الكتاب الذي أقرّته وزارة التربية.

ولمعالجة مشكلات كتب تعليم اللغة العربية، لقد أجريت دراسات كثيرة، سواء كانت في العالم الملايوي أم في غيره. ونظرا لأهمية دور الكتب في عملية تعليم وتعلم اللغة العربية بصفة عامة وفي ميدان

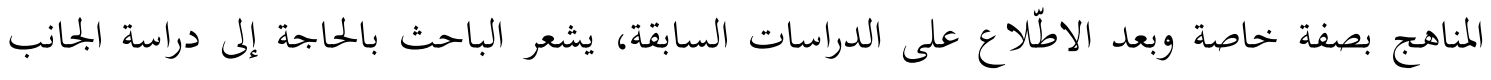
الذي لم يتم فحصه في الدراسات السابقة، وهي أنشطة التواصل الشفوي، وحيث أن سلسلة العربية

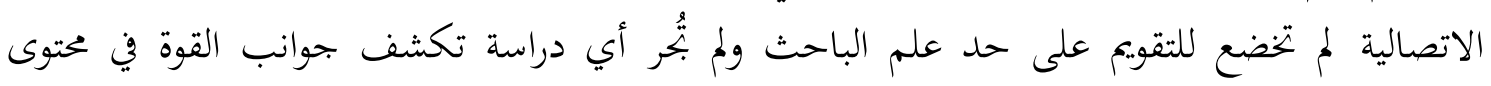
أنشطتها المتعلقة بالتواصل الشفوي-السمعي، ليتم دعمها وجوانب القصور ليتم تلافيها.

\section{منهجية البحث}

اتبع هذا البحث المنهج الكمي واستخدم الاستبانة للتعرف على آراء المعلمين عن أنشطة التواصل الشفوي-السمعي الموجودة في سلسلة كتب العربية الاتصالية، وتم تحليل البيانات الاستقصائية باستعان المان المعالمة الاحصائية المعروفة ب SPSS. اشتمل بحتمع الدراسة جميع معلمي اللغة العربية في المدارس الثانوية

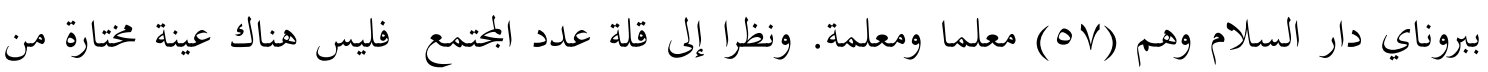
المحتمع في هذه الدراسة، فابلمتمع ككل هم العينة في هذه الدراسة. وتكوّنت الاستبانة للدراسة الحالية من مقدمة تحتوي المعلومات عن هدف البحث وعنوانه والملاحظة المهمة كما تحتوي الشكر للمستجيبين

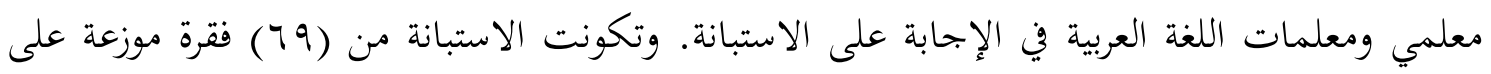
(ع) أقسام وهي قسم معلومات أساسية يتضمن أسئلة عن المعلومات الشخصية للمجيب مثل :المرحلة

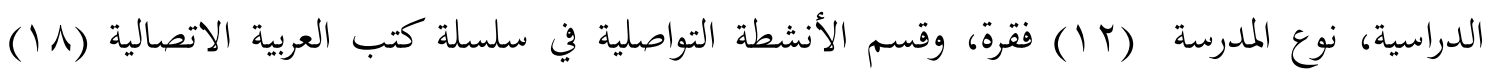
فقرة، وقسم مدى فعالية الأنشطة الموجودة في سلسلة كتب العربية الاتصالية في تنمية مهارات التواصل الشفوي-السمعي (T T) فقرة، وقسم أنواع أنشطة التواصل المتوفرة في السلسلة (YV) فقرة.

تمّ التحقق من صدق الأداة حيث عرضت الاستبانة على ستة (7) محكمين متخصصين أكاديميين في اللغة العربية والميدان التربوي، ثم عرض الباحث الاستبانة المعدّلة من قبل المحكمين على بحتمع الدراسة

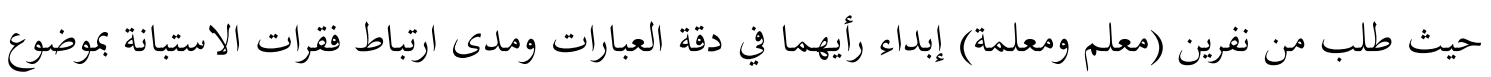

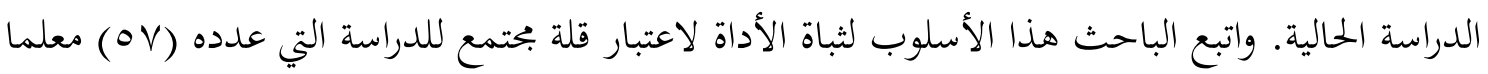

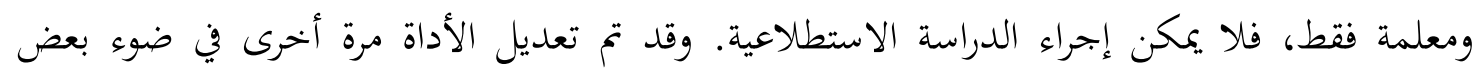


الملحوظات على المحتوى والصياغة، لتصبح جاهزة للتطبيق النهائي بعد الاطمئنان إلى حد كبير على صدقها.

\section{نتائج البحث}

حُلّلت آراء المعلمين عن الأنشطة الموجودة في سلسلة كتب العربية الاتصالية وفقا لثلاثة بحالات.

وابلحالات الثلاثة هي: الأنشطة التواصلية في سلسلة كتب العربية الاتصالية، مدى فعالية الأنشطة الموجودة

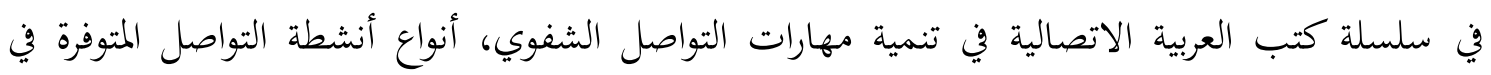
السلسلة، وتمّ نقل إجابات المشاركين في الاستبيان إلى برنامج SPSS للحصول على النسب المبل المئوية والتكرارات والمتوسطات الحسابية والانحرافات المعيارية لجميع الفقرات والبحالات. وفيما يلي تفصيل النتائج:

\section{الأنشطة التواصلية في سلسلة كتب العربية الاتصالية}

هدف هذا القسم إلى تعرّف على الأنشطة التواصلية في سلسلة كتب العربية الاتصالية. وبعد إجراء عمليات التحليل الاحصائي اللازمة وتطبيق إجراءات الدراسة على الاستبانة، فقد تم الحصول على النتائج وفيما يلي عرضها:

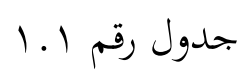

التكرارات والنسب المئوية والمتوسطات الحسابية الاخرافات المعيارية وترتيبها تنازليا لرأي عينة الدراسة حول الأنشطة التواصلية في سلسلة كتب "العربية الاتصالية

\begin{tabular}{|c|c|c|c|c|c|}
\hline \multirow[t]{4}{*}{ الدرجة } & الانحرا & المتوس & النسب المئوية (\%\%) & الفقرة & J \\
\hline & ف & $b$ & & & \\
\hline & المعيار & الحسا & التكرارات (f) & & \\
\hline & ي (SD) & بي & & & \\
\hline
\end{tabular}




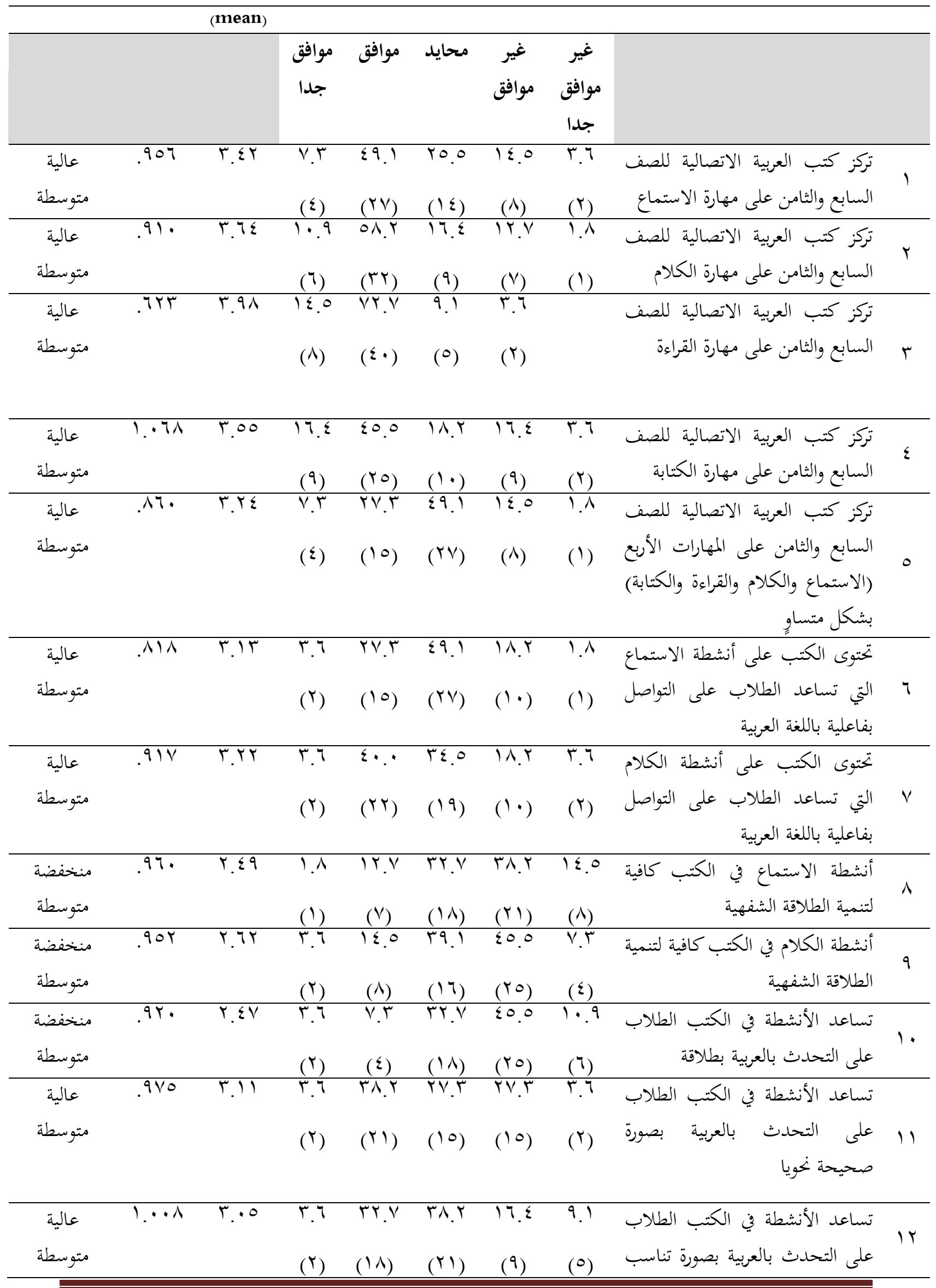




\begin{tabular}{|c|c|c|c|c|c|c|c|c|c|}
\hline & & & & & & & & كلّ موقف & \\
\hline متوسطة & 1.41 & $T . Y Y$ & $(r)$ & $\begin{array}{l}\sum Y . \Gamma \\
(Y \uparrow) \\
\end{array}$ & 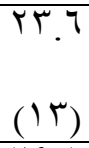 & $\begin{array}{l}1 \wedge . Y \\
(1 \cdot) \\
\end{array}$ & $\begin{array}{l}. r \\
(\varepsilon) \\
\end{array}$ & تحتوي الكتب على أنشطة تساعد & r \\
\hline متوسطة & $.9 \lambda r$ & $\Gamma .11$ & $(r)$ & $\begin{array}{l}\xi 1 . \wedge \\
(Y T)\end{array}$ & $\begin{array}{l}\text { rq.1 } \\
(17)\end{array}$ & $\begin{array}{l}r \cdot \cdot \\
\text { (1) }\end{array}$ & $\begin{array}{l}0.0 \\
(\mu)\end{array}$ & تساعد المحادثات في الكتب الطلاب التدرب على التحدّث الحر & $1 \leq$ \\
\hline متوسطة &.$\wedge N 4$ & $T . \leqslant V$ & T. 7 & 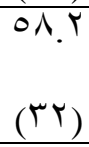 & $\begin{array}{l}r \cdot \cdot \\
(11) \\
\end{array}$ & $\begin{array}{l}1 \wedge . Y \\
(1 \cdot) \\
\end{array}$ & $\begin{array}{l}- \\
- \\
\end{array}$ & تدعم الصور الموجودة في الكتب & 10 \\
\hline منخفضة & $1 \ldots 9$ & T.TY & $r .7$ & $\begin{array}{l}1 \leqslant 0 \\
(\wedge)\end{array}$ & (19) & (19) & $\begin{array}{l}\text { IY.V } \\
(V)\end{array}$ & 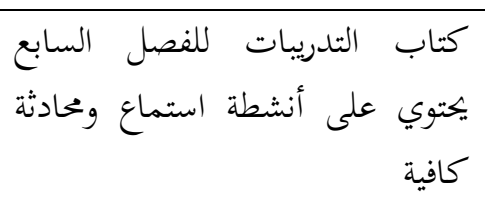 & 17 \\
\hline منتوسطة & ATO & T.T\& & (1) & $\begin{array}{l}1 \cdot .9 \\
(7)\end{array}$ & (YT) & $\begin{array}{l}\xi \cdot \cdot \cdot \\
(Y Y)\end{array}$ & $\begin{array}{l}0.0 \\
(\Gamma)\end{array}$ & كتاب كتية التدريبات للفصل الثامن & IV \\
\hline متوسطة & 919 & $r . \leqslant 0$ & 0.0 & $\begin{array}{l}\text { OY.Y } \\
(Y q)\end{array}$ & $(17)$ & $(\varepsilon)$ & $\begin{array}{l}0.0 \\
(\Gamma)\end{array}$ & الموضلاب من التواصل بالعربية شفهيات في الكتب تمُن & 11 \\
\hline متوسطة & $.7 Y$ & $r .1 T$ & & & & & & المتوسط العام & \\
\hline
\end{tabular}

ويتضح من الجدول أن بحموع المتوسط الحسابي لتقديرات المعلمين التقويمية لقسم الأنشطة

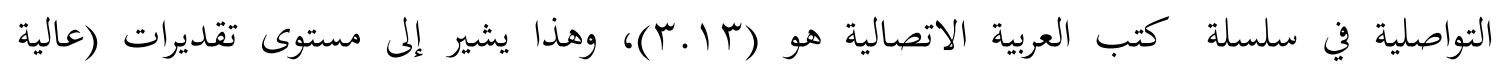
متوسطة). كما نستخلص منه أن درجات التقدير التقيمي لفقرات الاستبانة لهذا القسم كان بين تقديرات عالية متوسطة ومنخفضة متوسطة، وكان المتوسط الحسابيّ للاستبانة بتقدير تقويمي عالية متوسطة أي الحاجة إلى تنمية وتطوير الأنشطة التواصلية في سلسلة كتب العربية الاتصالية للصف السابع والثامن للمدارس الثانوية ببروناي دار السلام

\section{مدى فعالية الأنشطة الموجودة في سلسلة كتب العربية الاتصالية في تنمية مهارات التواصل} الشفوي-السمعي

هدف هذا القسم إلى تعرّف على مدى فعالية الأنشطة الموجودة في في سلسلة كتب العربية الاتصالية في تنمية مهارات التواصل الشفوي-السمعي. وبعد إجراء عمليات التحليل الاحصائي اللازمة وتطبيق إجراءات الدراسة على الاستبانة، فقد تم الحصول على النتائج وهي: 


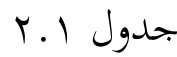

التكرارات والنسب المئوية والمتوسطات الحسابية الاخرافات المعيارية وترتيبها تنازليا لرأي عينة الدراسة حول مدى فعالية الأنشطة الموجودة في سلسلة كتب العربية الاتصالية في تنمية مهارات التواصل الشفوي

باستخدام الأنشطة التواصلية الموجودة في الكتب يستطيع المتعلمون أن:

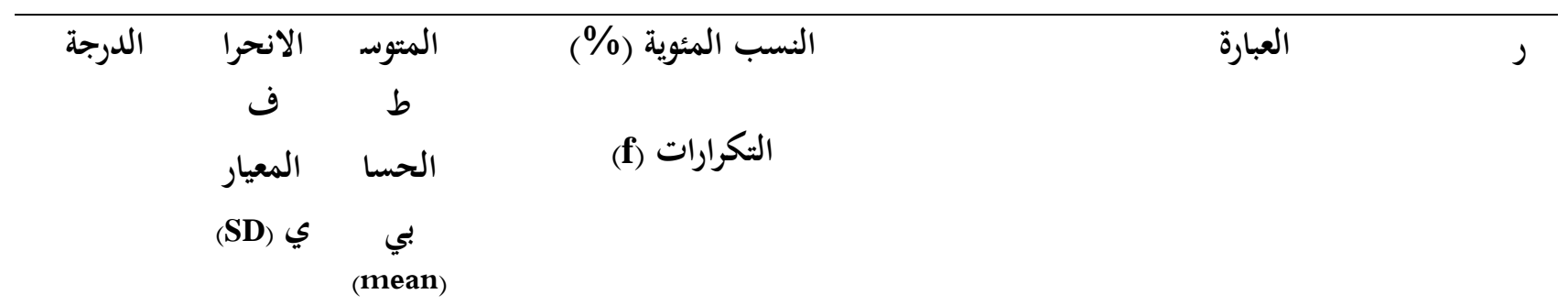

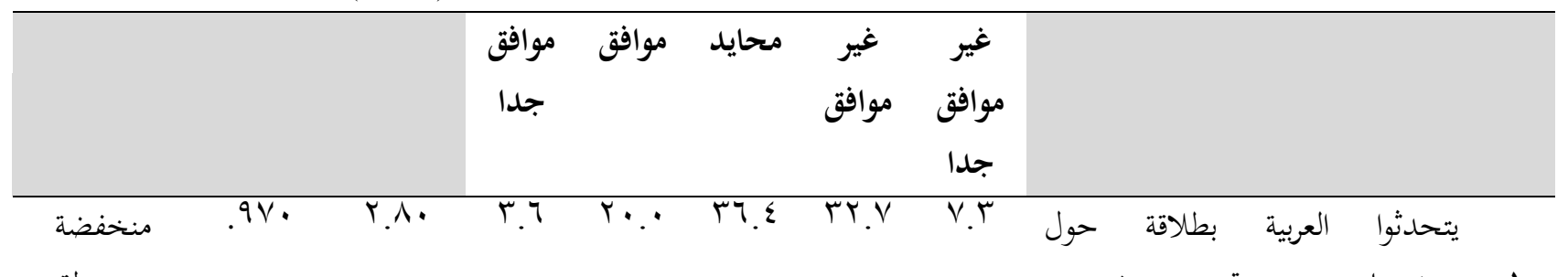
متوسطة
$(r$
(1) (Y.) (।^)
(飞)

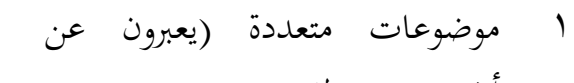

\begin{tabular}{|c|c|c|c|c|c|c|c|c|c|}
\hline & & & & & & & & أنفسهم بسهولة) & \\
\hline منخفضة & AT & T. $\leqslant \varepsilon$ & T.7 & 1.1 & rฯ.人 & $\varepsilon \vee . \Gamma$ & 9.1 & يقوموا بمحادثة هاتفية باللغة العربية & $r$ \\
\hline 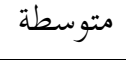 & & & $(r)$ & (1) & $\left(Y_{1}\right)$ & $\left(Y^{\top}\right)$ & (0) & & \\
\hline منخفضة & VAr. & T.TY & $1 \leqslant 0$ & $1 \leqslant .0$ & r..ร & $\leqslant 0.0$ & $r .7$ & يتبادلوا المعلومات باللغة العربية & س \\
\hline
\end{tabular}

\begin{tabular}{|c|c|c|c|c|c|c|c|c|}
\hline متوسطة & & & $(\wedge)$ & $(\wedge)$ & $(Y \cdot)$ & 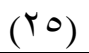 & $(r)$ & \\
\hline منخفضة & $I . T V$ & T.VT & T.7 & YI.A & $\overline{r V . r}$ & r^.Y & 9.1 & يعبّروا عن أنفسهم باللغة العربية دون \\
\hline 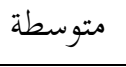 & & & $(r)$ & $(1 Y)$ & $(10)$ & $\left(Y^{\prime}\right)$ & ( 1$)$ & استخدام اللغة الملايوية \\
\hline منخفضة & .AYI & T.TO & 1.1 & T.7 & $r \leqslant .0$ & $\varepsilon V . \Gamma$ & IY.V & يتحدثوا بالجودة التي يكتبون بما \\
\hline
\end{tabular}

\begin{tabular}{|c|c|c|c|c|c|c|c|c|c|}
\hline 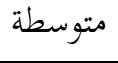 & & & ( $)$ & $(Y)$ & $(19)$ & $(Y 7)$ & $(\vee)$ & & \\
\hline عالية الية & $1 . . \leq \leqslant$ & $T . T V$ & 9.1 & $\varepsilon \cdot \cdot$ & Tr.T & rT. & T.7 & يجيبوا عن أسئلة تعتمد على نص & 7 \\
\hline متوسطة & & & (0) & $(Y Y)$ & $(1 T)$ & $(1 / T)$ & $(r)$ & استماع ملائم & \\
\hline منخخفضة & 919 & T.00 & 1.1 & TY.V & $r \leqslant .0$ & $\varepsilon \cdot \cdot$ & 1.99 & يقدّموا ملخّصصا أو تعليقا شفهيا على & V \\
\hline
\end{tabular}




\begin{tabular}{|c|c|c|c|c|c|c|c|c|c|}
\hline متوسطة & & & (1) & $(\mathrm{V})$ & $(19)$ & $(Y Y)$ & (7) & موضوع ما & \\
\hline متخخفة متوسطة & . ATr & Y.11 & - & (1) & $(19)$ & $\begin{array}{l}\text { T.. } \\
(Y \cdot)\end{array}$ & $\begin{array}{l}\text { YV.T } \\
(10)\end{array}$ & 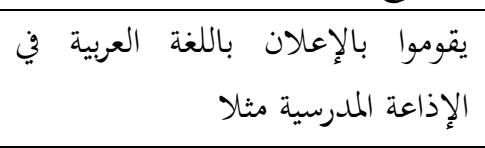 & $\wedge$ \\
\hline منتخفضة & $.9 Y$. & T.T & - & $\begin{array}{l}1 \cdot .9 \\
(7) \\
\end{array}$ & $\begin{array}{l}r 9.1 \\
(17) \\
\end{array}$ & $\begin{array}{l}\varepsilon \cdot \cdot \cdot \\
(Y Y)\end{array}$ & $\begin{array}{l}r \cdot \cdot \\
(11)\end{array}$ & بعلكّقوا على صورة أو فيلم أو مباراة & 9 \\
\hline منتخفضة & .9 .8 & ז.ro & - & $\begin{array}{l}9.1 \\
(0)\end{array}$ & $\begin{array}{l}r . \varepsilon \\
(Y \cdot)\end{array}$ & (19) & $\begin{array}{l}r \cdot \cdot \\
(11)\end{array}$ & 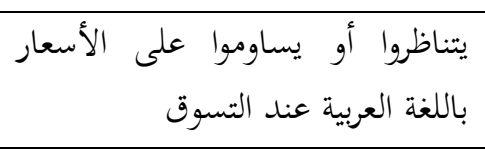 & 1. \\
\hline منتخفضة & $1.7 V$ & T.07 & (1) & $\begin{array}{l}\text { (II.人 } \\
(1 Y)\end{array}$ & 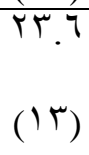 & 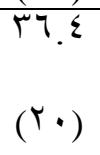 & $\begin{array}{l}17.5 \\
(9)\end{array}$ & يقوموا بعمل مقابلة شخصية & 11 \\
\hline متخفضة & $1 . \leqslant r$ & Y.TE & (1) & $\begin{array}{l}r \cdot \cdot \\
(11)\end{array}$ & $\begin{array}{l}r \leqslant .0 \\
(19)\end{array}$ & $\begin{array}{l}\text { YV.r } \\
(10)\end{array}$ & $\begin{array}{l}17.5 \\
(9)\end{array}$ & يقوموا بتمثيل الأدوار المسرحية باللغة & ir \\
\hline منخفضضة & .79 & 1.00 & & & & & & المتوسط العام & \\
\hline
\end{tabular}

يتضح من الجحدول أن بحموع المتوسط الحسابي لتقديرات المعلمين التقويمية لقسم مدى فعالية

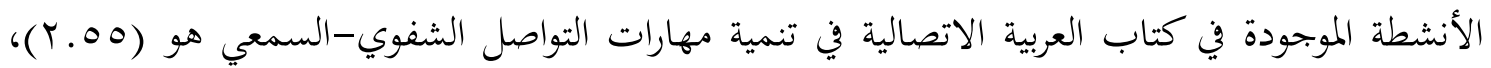
وهذا يشير إلى مستوى تقديرات (منخفضة متوسطة). ويلاحظ من هذا الجحدول أيضا أن معظم تقديرات

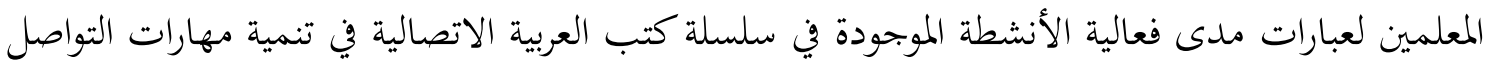
الشفوي-السمعي (II فقرة) من بحموع الفقرات (T I ) فقرة كانت بدرجة منخفضة متوسطة، وهذه

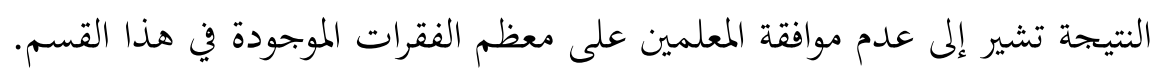

\section{أنواع أنشطة التواصل المتوفرة في كتب العربية الاتصالية}

هدف هذا القسم إلى تعرّف على أنواع أنشطة التواصل المتوفرة في سلسلة كتب العربية الاتصالية. وبعد

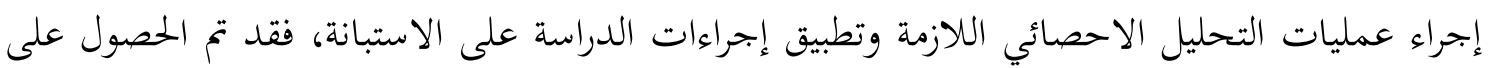

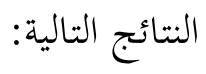

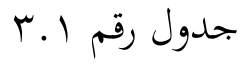

التكرارات والنسب المئوية والمتوسطات الحسابية الانحرافات المعيارية وترتيبها تنازليا لرأي عينة الدراسة حول أنواع أنشطة التواصل المتوفرة في سلسلة كتب العربية الاتصالية 


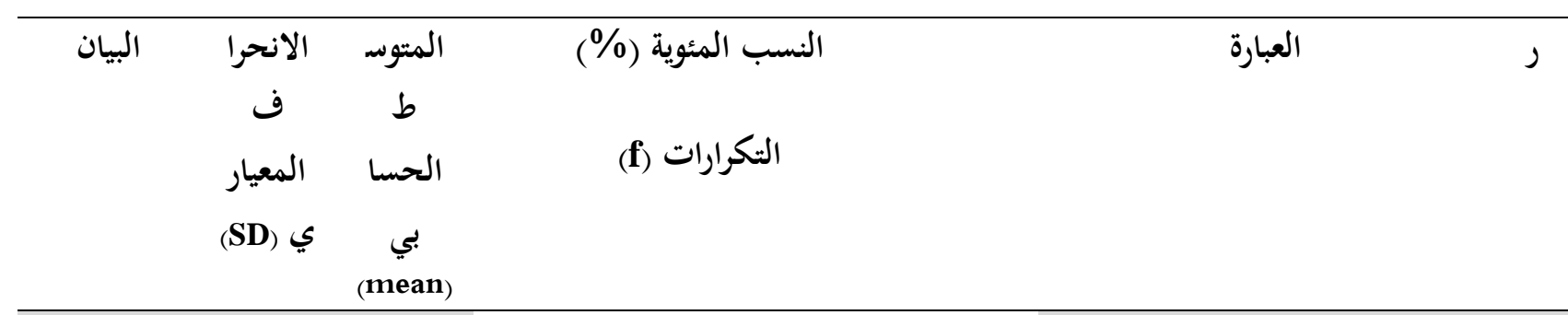

\begin{tabular}{|c|c|c|c|c|c|c|c|c|c|}
\hline & & & موافق & موافق & محايد & غوافق & غوافق & مواصلة جدول ع.7. & \\
\hline متوسطة & $.9 \leq r$ & $r . \cdot \varepsilon$ & $\begin{array}{l}1.1 \\
\text { (1) }\end{array}$ & $\begin{array}{l}\text { YY.V } \\
(1 \wedge)\end{array}$ & $\begin{array}{l}\varepsilon \cdot \cdot \\
(Y r)\end{array}$ & $\begin{array}{l}1 \wedge . r \\
(1 \cdot)\end{array}$ & $\begin{array}{l}V . r \\
(\varepsilon)\end{array}$ & الفصل & 1 \\
\hline منتخفضة & $1 . T \leq$ & $r .9 r$ & - & $\begin{array}{l}r \Lambda . r \\
(Y I) \\
\end{array}$ & $(10)$ & 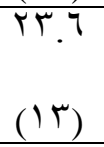 & $\begin{array}{l}1 \cdot .9 \\
(7) \\
\end{array}$ & 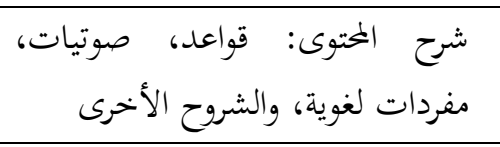 & r \\
\hline متوسطة & $9 Y \xi$ & $r .1 T$ & - & 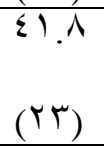 & $\begin{array}{l}\text { rฯ. } \\
(Y \cdot)\end{array}$ & $\begin{array}{l}1 \leqslant 0 \\
(\wedge)\end{array}$ & $(\varepsilon)$ & 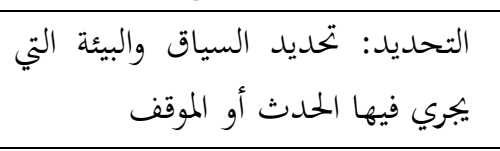 & $r$ \\
\hline متوسطة & $.97 \varepsilon$ & r.11 & $\begin{array}{l}1 . \wedge \\
(1)\end{array}$ & $\begin{array}{l}\leqslant 0.0 \\
(Y 0)\end{array}$ & $\begin{array}{l}\text { (10) } \\
\text { rV.r }\end{array}$ & $\begin{array}{l}r \cdot \cdot \\
(11)\end{array}$ & $\begin{array}{l}0.0 \\
(\Gamma)\end{array}$ & 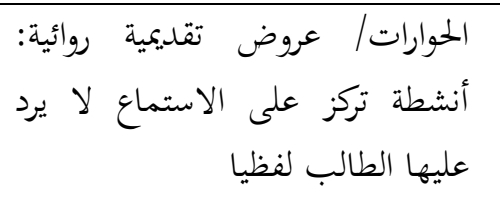 & $\varepsilon$ \\
\hline متوسطة & 1.1 .0 & T.Yร & $\begin{array}{l}. \Gamma \\
(\varepsilon)\end{array}$ & $\begin{array}{l}\text { \&Y. } \\
(Y \varepsilon)\end{array}$ & $\begin{array}{l}\text { (IT) } \\
\text { rT. }\end{array}$ & $\begin{array}{l}17.5 \\
(9)\end{array}$ & $\begin{array}{l}9.1 \\
\text { (0) }\end{array}$ & علئهم الإملاء: يكتب الطلاب نصوصا تملى & 0 \\
\hline متخوسطة & $1 . V Y$ & T.TV & $\begin{array}{l}1 . \wedge \\
(1) \\
\end{array}$ & $(1 T)$ & $\begin{array}{l}r \cdot .9 \\
(1 \vee)\end{array}$ & $\begin{array}{l}\text { T.T } \\
(10) \\
\end{array}$ & $\begin{array}{l}17.5 \\
(9)\end{array}$ & ألعاب تستخدم: أغاني / أناشيد، نلأنشطة التمهيدية، & 7 \\
\hline منخفضطة & 901 & T.A. & (1) & $(\mathrm{I})$ & $\begin{array}{l}\varepsilon \cdot \cdot \\
(T+)\end{array}$ & $\begin{array}{l}\text { (10) } \\
\text { TV.T }\end{array}$ & $\begin{array}{l}9.1 \\
(0)\end{array}$ & 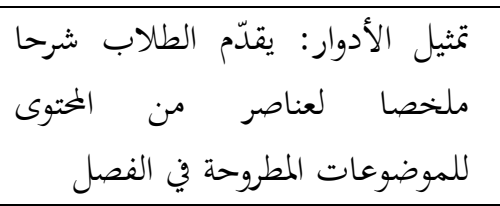 & v \\
\hline متوسطة & .94. & T. $\leqslant q$ & $\begin{array}{l}0.0 \\
(1)\end{array}$ & $\begin{array}{l}07.5 \\
\left(\Gamma^{0}\right)\end{array}$ & $\begin{array}{l}\text { ro.0 } \\
(1 \varepsilon)\end{array}$ & $\begin{array}{l}\text { V.r } \\
(\varepsilon)\end{array}$ & $\begin{array}{l}0.0 \\
(1)\end{array}$ & 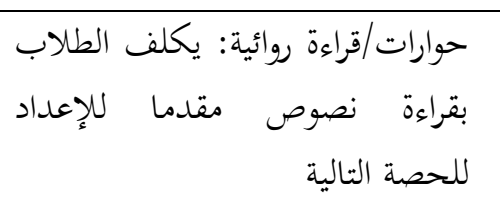 & $\wedge$ \\
\hline متوسطة & VIr & $r . V I$ & $\begin{array}{l}r .7 \\
(Y)\end{array}$ & $\begin{array}{l}\text { VY.V } \\
(\varepsilon \cdot)\end{array}$ & $\begin{array}{l}17.5 \\
(9)\end{array}$ & $\begin{array}{l}0.0 \\
(\Gamma)\end{array}$ & $\begin{array}{l}1.1 \\
\text { (1) }\end{array}$ & من القراءة بصوت عال: نصوص يطلب قراء & 9 \\
\hline متوسطة & .091 & $r .90$ & $\begin{array}{l}1 \cdot .9 \\
(7)\end{array}$ & $(\varepsilon r)$ & $(0)$ & $(r)$ & $\begin{array}{l}- \\
- \\
\end{array}$ & أسئلة المعلم شفهيال والجواب: يجيب الطلاب عن & • \\
\hline متوسطة &.$\wedge 97$ & $r .01$ & $(\varepsilon)$ & $\begin{array}{l}7 \cdot \cdot \\
(\mu r)\end{array}$ & (1) & $(0)$ & $\begin{array}{l}r .7 \\
(T)\end{array}$ & كلمات بأخرى تحمل معنى مقاربا تكرار، استبدال & 1 \\
\hline عالية &.$V T V$ & r.VI & V.r & 70.0 & $1 \Lambda . r$ & 9.1 & & تدريب بالمفهوم: يستجيب الطلاب & Ir \\
\hline
\end{tabular}




\begin{tabular}{|c|c|c|c|c|c|c|c|c|c|}
\hline متوسطة & & & $(\varepsilon)$ & $(\Gamma \tau)$ & $(1 \cdot)$ & $(0)$ & - & لمعلومات مختلفة ذات مدلول كمرجعية & \\
\hline متوسطة & $1.1 \leq$ & 5.17 & $\begin{array}{l}1.1 \\
(1)\end{array}$ & $\begin{array}{l}\sum V \cdot T \\
(Y \tau)\end{array}$ & $\begin{array}{l}(1 T) \\
T . T\end{array}$ & $\begin{array}{l}r \cdot \cdot \\
(11)\end{array}$ & $\begin{array}{l}\text { V.r } \\
(\varepsilon)\end{array}$ & عرواية القصص: يستمع الطلاب إلى & Tr \\
\hline متوسطة &.$\wedge \wedge \mu$ & $r .1 T$ & (1) & $\begin{array}{l}r \leqslant 0 \\
(19)\end{array}$ & $(Y \Sigma)$ & $\begin{array}{l}1 \leqslant 0 \\
(\wedge)\end{array}$ & $\begin{array}{l}0.0 \\
(\Gamma)\end{array}$ & نقل البيانات بينما يستمعون إلى وصفوم الطلاب بملى & $1 \varepsilon$ \\
\hline متوسطة & 911 & T.Y & $\begin{array}{l}. T \\
(\varepsilon)\end{array}$ & $\begin{array}{l}\text { TY. } \\
(\mathrm{N}) \\
\end{array}$ & 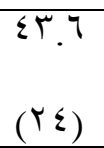 & $\begin{array}{l}9.1 \\
(0)\end{array}$ & $\begin{array}{l}\text { V.r } \\
(\varepsilon) \\
\end{array}$ & تلخيص يلقيه ابلميجاز. يستمع الطلاب إلى ألى الطلاب الطلاب & 10 \\
\hline متوسطة & AVO & $\Gamma .01$ & $\bar{V} . r$ & 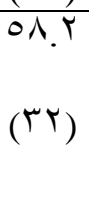 & 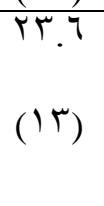 & $(\varepsilon)$ & (T) & 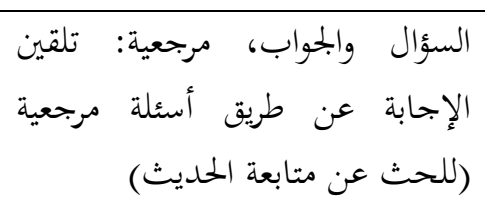 & 17 \\
\hline متوسطة & VOV & T.TY & $\begin{array}{l}\text { V.T } \\
(\varepsilon)\end{array}$ & $\begin{array}{l}0 \leqslant 0 \\
(r \cdot)\end{array}$ & $(\backslash \wedge)$ & $\begin{array}{l}r .7 \\
(r)\end{array}$ & $\begin{array}{l}1 . \wedge \\
\text { (1) }\end{array}$ & الرواية أو حوار بالتلميحات: يستعين & iv \\
\hline متوسطة & 974 & T.Y & $\begin{array}{l}0.0 \\
(1)\end{array}$ & $\begin{array}{l}\text { rA. } \\
\left(Y^{\prime}\right)\end{array}$ & $\begin{array}{l}\text { T.. } \\
(Y \cdot)\end{array}$ & $\begin{array}{l}1 \leqslant 0 \\
(\wedge)\end{array}$ & $\begin{array}{l}0.0 \\
(1)\end{array}$ & 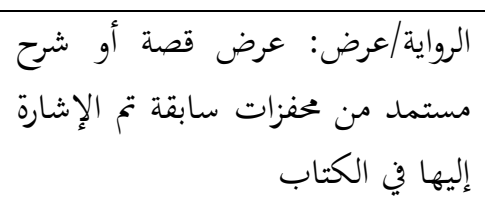 & 11 \\
\hline منخفضة & $.9 Y \wedge$ & 7.91 & $\begin{array}{l}\text { r. } \\
(4)\end{array}$ & $\begin{array}{l}r \cdot \cdot \\
(11)\end{array}$ & $\begin{array}{l}\Sigma V . r \\
(Y \top)\end{array}$ & $\begin{array}{l}\text { YI.A } \\
(1 Y)\end{array}$ & $\begin{array}{l}\text { V.r } \\
(\varepsilon)\end{array}$ & موضهة من الشحز الذهني: مناقشات حرة غير & 19 \\
\hline متوسطة & $9 \leqslant \wedge$ & $r .9$ & $\begin{array}{l}1 . \wedge \\
(1)\end{array}$ & $\begin{array}{l}r\urcorner . \xi \\
(T \cdot) \\
\end{array}$ & $\begin{array}{l}r \wedge . r \\
\left(Y I^{\prime}\right)\end{array}$ & $\begin{array}{l}17.5 \\
(9)\end{array}$ & $\begin{array}{l}V . r \\
(\varepsilon)\end{array}$ & تمثيل الأدوار: تمثيل حر نسبيا لأدوار & $r$. \\
\hline متوسطة & 1.77 & T.YY & $\begin{array}{l}0.0 \\
(1)\end{array}$ & $\begin{array}{l}\varepsilon r .7 \\
(Y \leq)\end{array}$ & $(10)$ & (1) & $\begin{array}{l}9.1 \\
(0)\end{array}$ & الألعاب: لعبة لوحة الترتيب لتكوين & YI \\
\hline منتخفضة & $1 \ldots$ & r.. & $\begin{array}{l}\text { (T) } \\
\text { r. }\end{array}$ & $\begin{array}{l}r 9.1 \\
(17)\end{array}$ & $\begin{array}{l}\varepsilon \cdot \cdot \cdot \\
(Y T)\end{array}$ & $\begin{array}{l}1 \wedge .1 \\
(1 \cdot)\end{array}$ & $\begin{array}{l}9.1 \\
(0)\end{array}$ & 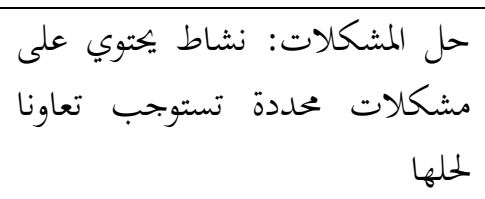 & Yr \\
\hline منتخفضة & $1 . I K Y$ & r.97 & $\begin{array}{l}\text { r. } \\
(r)\end{array}$ & $\begin{array}{l}r \leqslant .0 \\
(19)\end{array}$ & $\begin{array}{l}r \cdot .9 \\
(1 v)\end{array}$ & $\begin{array}{l}17.5 \\
(9)\end{array}$ & $\begin{array}{l}1 \leqslant 0 \\
(\wedge)\end{array}$ & 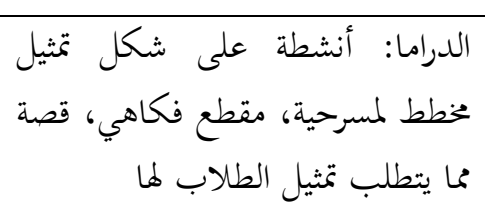 & r \\
\hline متوسطة & $.99 \leqslant$ & r. Yq & $\begin{array}{l}r . T \\
(4)\end{array}$ & $\begin{array}{l}\sum 9.1 \\
(Y V)\end{array}$ & $\begin{array}{l}\text { YV.r } \\
(10)\end{array}$ & $\begin{array}{l}\text { IY.V } \\
(\mathrm{V})\end{array}$ & $\begin{array}{l}V . r \\
(\varepsilon)\end{array}$ & علماكلى مواقف: الأنشطة في الكتاب تبنى & $r \varepsilon$ \\
\hline منخفضة & $1.5 \leq \varepsilon$ & T.90 & - & $r 7 . \xi$ & r. & IT.V & $1 \leqslant 0$ & المقابلة: مهام موجهة لطلاب & ro \\
\hline
\end{tabular}




\begin{tabular}{|c|c|c|c|c|c|c|c|c|c|}
\hline متوسطة & & & & $(Y \cdot)$ & $(Y \cdot)$ & $(\mathrm{V})$ & $(\wedge)$ & للحصول على المعلومات من بعضهم & \\
\hline متوسطة & $.9 V \cdot$ & $r .10$ & $\begin{array}{l}0.0 \\
(1)\end{array}$ & $\begin{array}{l}\text { OY.V } \\
(Y T)\end{array}$ & $\begin{array}{l}\text { Y9.1 } \\
(11)\end{array}$ & $\begin{array}{l}V_{. T} \\
(9)\end{array}$ & $\begin{array}{l}0.0 \\
(\varepsilon)\end{array}$ & للملاقشة: مواد مقدمة في الكتاب يقوموا بمناقشتها & rt \\
\hline منتخفضة & .10. & t.r人 & - & $\begin{array}{l}9.1 \\
(0)\end{array}$ & (19) & 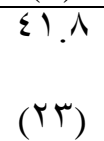 & $1 \leqslant 0$ & للملاطرة: مواد مقدمة في الإجراء مناظرات حولهاب & rV \\
\hline متوسطة & $\because$ Or & 5.19 & & & & & & المتوسط العام & \\
\hline
\end{tabular}

يدل الجحدول السابق على أن بحموع المتوسط الحسابي لتقديرات المعلمين التقويمية لقسم أنواع

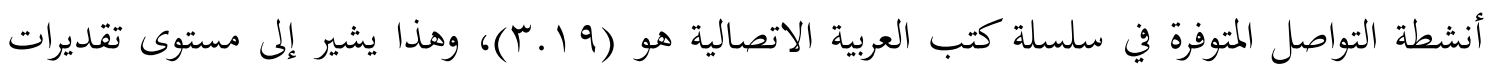
(عالية متوسطة). وتتوسط درجات التقدير التقويمي لفقرات الاستبانة لهذا القسم كان بين تقديرات عالية متوسطة ومنخفضة متوسطة، وكان المتوسط الحسابيّ عالية متوسطة، وهذا يعني الحاجة إلى تنمية وتطوير أنواع الأنشطة التواصلية في سلسلة كتب العربية الاتصالية للصف السابع والثامن للمدارس الثانوية ببروناي دار السلام.

\section{خلاصة البحث}

من خلال نتائج البحث تبين أن هناك عدة آثار مرتبة على الكتب الحالية. الأثار الأول هو أن هناك حاجة ماسة لتغيير معظم الموضوعات والأنشطة الموجودة في الكتب الحالية لتكون مناسبة مع مرحلة المبتدئين. ويجب تغيير الأنشطة الموجودة لتكون أكثر ملبية لحاجة المتعلمين اليومية وثقافاتم البروناوية، وأن تكون أكثر بساطة وأكثر جاذبية وإثارة للاهتمام. والأثر الثاني هو تقديم الاهتمام أكثر في تنمية مهارة

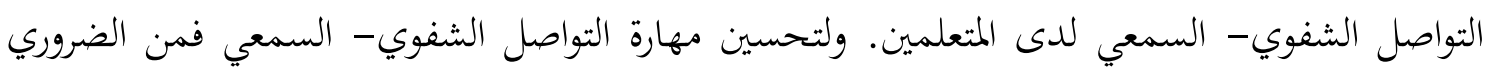
اشتمال الكتب على الأنشطة المتنوعة التي تدعم تنمية التواصل الشفوي- السمعي عندهم، وأن يرتبط التقويم الفصلي والامتحانات بهدف ترقية التواصل الشفوي- السمعي بين المتعلمين.

المصادر والمراجع 


$$
\begin{aligned}
& \text { فغيران الحاج شهاب الدين، فغيران حاجه سلمية بنت فغيران الحاج شهاب الدين. (999 1). تعليم اللغة } \\
& \text { العربية في سلطنة بروناي دار السلام مشكلاته ومعالجتها ـ بحث مقدم إلى كلية الدراسات } \\
& \text { الإسلامية بجامعة بروناي دار السلام للحصول على درجة الماجستير. } \\
& \text { محمود كامل الناقة ورشدى أحمد طعيمة. (ب191 (). الكتاب الأساسي لتعليم الغة العربية للناطقين } \\
& \text { بلغات أخرى إعداده - تحليله - تقويمه. مكة المكرمة. جامعة أم القرى. } \\
& \text { نور الهدى بنت عثمان (0 . ․ ץ)، تعليم اللغة اللعبية للناطقين بغيرها ؛ الكتاب الأساسي الجزء الأول } \\
& \text { دراسة تقويمية. رسالة الماجيتير. الجامعة الإسلامية العالمية ماليزيا } \\
& \text { الهاشمي، عبد الرحمن، محسن علي عطية (9 . . ب). تحليل محتوى مناهج اللغة العببية رؤية نظرية } \\
& \text { تطبيقية. عمان: دار الصفاء للنشر والتوزيع. }
\end{aligned}
$$

Gamal Abdul Nasir Zakaria, Salwa Mahalle, \& Aliff Nawi. (ץ-10). Kajian Amalan Pengajaran Guru Bahasa Arab Sekolah Menengah di Negara Brunei Darussalam. Q-jIE The Online Journal of Islamic Education, r( ) , r r $-\varepsilon \cdot$

Noorhayati Hashim ( $Y . .9$ ). Keberkesanan Kaedah Noor Dalam Pengajaran Dan P Pembelajaran Nahu Bahasa Arab. Tesis Doktor Falsafah Pendidikan. Kuala Lumpur:Universiti Malaya.

Norasfidah binti Haji Bakir. $(\uparrow \cdot 1 Y$ ). Pengajaran Bahasa Arab Asas: Pelaksanaannya di Sekolah-sekolah Rendah Ugama di Daerah Brunei Muara Kawasan Brunei II.

Disertasi Sarjana Muda. Brunei Darussalam: Fakulti Syariah, Kolej Universiti Perguruan Ugama Seri Begawan (KUPUSB).

Zohrabi, M., Sabouri, H., \& Behroozian, R. (r.1r). An evaluation of merits and demerits of Iranian first year high school English textbook. English Language

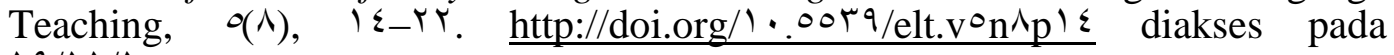
$19 / 11 / 10$. 